\title{
Study on the Efficacy of Sentinel Lymph Node Identification by Radionuclide Method (Tc 99) Versus Combined Method (Radionuclide and Vital Stain) in the Staging of Breast Carcinoma
} \author{
Oprea1, Andreea Păscutoi ${ }^{1}$, Adrian Naznean ${ }^{4}$, Simona Stolnicu ${ }^{5}$ \\ 1 Department of Surgery, University of Medicine and Pharmacy, Tîrgu Mureș, Romania \\ 2 Department of Nuclear Medicine, University of Medicine and Pharmacy, Tîrgu Mureș, Romania \\ 3 Department of Radiology, University of Medicine and Pharmacy, Tîrgu Mureș, Romania \\ 4 Department of Foreign Language, University of Medicine and Pharmacy, Tîrgu Mureș, Romania \\ 5 Department of Pathology, University of Medicine and Pharmacy, Tîrgu Mureș, Romania
}

Rareş Georgescu', Orsolya Bauer ${ }^{1}$, Marius Coroş¹, Rareş Barbat², Daniela Podeanu³ ${ }^{3}$ Adela

\section{CORRESPONDENCE}

Rareș Georgescu

Str. Gheorghe Marinescu nr. 38

540139 Tîrgu Mures, Romania

Tel: +40 265215551

E-mail: rares1geo@gmail.com

\section{ARTICLE HISTORY}

Received: 7 July, 2016

Accepted: 10 August, 2016
Orsolya Bauer, Marius Coroş, Rareş Barbat, Daniela Podeanu, Adela Oprea, Andreea Păscutoi, Adrian Naznean, Simona Stolnicu • Str. Gheorghe Marinesc nr. 38, 540139 Tîrgu Mureș, Romania. Tel: +40 265 215551

\begin{abstract}
Introduction: Sentinel node biopsy is the gold standard for axillary assessment of patients with breast cancer without axillary metastases on clinical and radiological examination. Internationally accepted biopsy methods currently use a radioactive tracer (Te) or different variations of vital stain, or the combination of the two. Due to the high cost of technical and organizational difficulty related to the radioactive material, as well as the disadvantages of using the vital stain method, great effort is being made to find alternative solutions. The aim of this study was to determine the effectiveness of the exclusive use of vital stain versus the radioactive isotope, and the need to use the combined method. A second goal was the comparative analysis of the radioactive method and intraoperative assessment of suspicious (non-sentinel) lymph nodes. Materials and methods: This article is based on a prospective nonrandomized study conducted on 69 patients with early breast cancer in whom the combined method was used (injection of radionuclide and methylene blue vital stain). The comparatively monitored parameters were the following: the total and mean number of excised sentinel lymph nodes, the number of metastatic ganglia revealed by the 2 methods, and the risk of understaging in case only one technique was used. Results: We excised 153 sentinel nodes identified by the radioisotope method. Of these only 56 were stained with methylene blue $(p<0.0001)$. We could also identify a significantly higher number of metastatic nodes with the aid of the radioactive method ( $p=$ 0.0049). Most importantly, a significant number of patients (57.14\%) who would have been declared node-negative using vital staining could only be properly staged using the radionuclide or the combined method. On microscopic examination of 35 non-sentinel lymph nodes, we found 3 lymph nodes with metastases, and in 1 case the metastases were found only in the non-sentinel lymph node. Conclusions: Given the risk of understaging, exclusive use of the vital stain method is not recommended, especially under the ASGO Z 00011 Protocol, since the more accurate determination of the number of metastatic sentinel lymph nodes in a patient
\end{abstract}




\begin{abstract}
influences the decision whether to perform lymphadenectomy or not. Using the combined method confers benefits only during the learning curve, in our database we found no stained nodes which were not radioactive. It is very important that the intraoperative stage uses the radioactive method and the intraoperative assessment of suspicious lymph nodes, because 35 non-sentinel lymph nodes were identified in our study, 3 of which had metastases, while in 1 case the metastases were in the non-sentinel lymph node.
\end{abstract}

Keywords: sentinel lymph node, radionuclide, vital stain

\section{INTRODUCTION}

Breast cancer is the most common malignancy, affecting females. ${ }^{1}$ Specific survival in breast cancer has improved significantly in recent decades, on the one hand thanks to early diagnosis due to screening programs, and, on the other hand, because of advances in the knowledge of tumor biology, which allowed the development of highly efficient targeted therapies. ${ }^{2}$

However, the role of surgery remains essential in multimodal treatment; efforts in this area are driven especially towards the development of less invasive techniques and fewer sequelae (both physical and psychological), and faster recovery. For a long time, surgical excision of lymph nodes played an important role in the surgical treatment of breast cancer. Its aim, on the one hand, is the local control of the neoplastic process, and, on the other, staging of the disease (important for adjuvant treatment decision).

Biopsy of sentinel lymph nodes (SLN) has become the standard procedure for assessing alternative axillary lymph damage in patients without clinically evident axillary metastases. $^{3}$ Initially, in cases with metastasis to the SLN, routine practice included Berg neck dissection of lymphatic levels I and II. A change of paradigm occurred after the publication of trial ASCGO Z00011, because avoiding lymphadenectomy was proved to be appropriate and safe in patients with positive SLN who met certain criteria. ${ }^{4}$ The AMAROS trial also showed overlapping results between axillary lymphadenectomy and irradiation in patients with a positive SLN without clinically evident lymph nodes, but with a lower morbidity in favor of radiation therapy. ${ }^{5}$

The application of these protocols requires a more accurate axillary assessment, which can prevent the risk of undertreating patients, especially in terms of oncology.

Initially, SLN was considered safe only in patients who had not received neoadjuvant therapy (chemotherapy or hormone therapy), because of the risk of inhomogeneous therapeutic response of the lymph nodes, but current studies show that the technique can be performed safely after the neoadjuvant therapy. ${ }^{6}$

The technique was initially based on injecting vital stain (methylene blue) and, subsequently, on injecting radionu- clide known for its lymphatic tropism, or on the combined method (methylene blue and radionuclides). ${ }^{7}$ Due to technical difficulties and costs related to the handling of radioactive substances (which involves cooperation between several specialists), alternative techniques were tested, such as exclusive use of the vital stain, or injection of lymphotropic magnetic powders. ${ }^{8,9}$

Previously published studies demonstrate the viability of exclusive use of the vital stain especially in underdeveloped countries where the radionuclide technique is unavailable. ${ }^{10}$ Other studies, however, show that this method is not very efficient in detecting SLN and, moreover, there is no real advantage in associating the vital stain method with the radioactive one. ${ }^{11-13}$

The aim of this paper was to evaluate the effectiveness of using radionuclide stain exclusively compared to the combined radionuclide method (vital stain and radioactive element) in SLN identification and correct and efficient staging of breast carcinoma. A second goal was the comparative analysis of the radioactive method and intraoperative assessment of suspicious lymph nodes, since there are few studies addressing this issue.

\section{MATERIALS AND METHODS}

We conducted a nonrandomized prospective study on a total of 69 cases of early breast cancer operated in the Tîrgu Mureș Surgical Clinic I between 2012-2015. Preoperative breast cancer was confirmed by ultrasound-guided biopsy in all patients. All patients underwent clinical, ultrasound, and preoperative mammography evaluation to highlight the presence or absence of suspicious lymph nodes. In patients whose imaging evaluation revealed suspicious lymph nodes, ultrasound-guided biopsy was performed with $18 \mathrm{G}$ needle for histopathological confirmation of lymph node metastases. Patients in whom the presence of lymph node metastases was confirmed were excluded from the study and underwent lymphadenectomy. None of the 69 patients included in this study showed suspicious lymph nodes in the clinical and/or radiological evaluation. In order to identify the sentinel lymph node, all patients 
included in this study were subjected to the radioactive method (radioisotope Tc 99) and to the combined method using both the vital stain (methylene blue) method, as well as the injection of radioisotope Tc 99. Two milliliters of radioactive substance (technetium Tc $99 \mathrm{~m}$ nanocolloid) were injected subcutaneously on the day of surgery in the Department of Nuclear Medicine. There are two variants regarding injecting, namely, periareolar or peritumoral injection. We used the combined technique of periareolar injection in 4 cardinal points and peritumoral injection in all the patients included in our study. Injection of radionuclides is followed by lymphoscintigraphy, which reveals the location of the sentinel lymph node. Intraoperative identification of the sentinel node is performed using the Geiger-Müller probe, with which we can identify the point of maximum radioactivity. This method was performed in all the patients of our study group.

Injecting methylene blue vital stain was done prior to the surgery; $2 \mathrm{~mL}$ were injected at the four-periareolar sites, intradermally. This method was performed in all the patients of our group.

By definition, lymph nodes that stain blue through the vital stain method are considered positive, while the hot lymph node is the one that captured radioactive isotope after injecting the radioactive substance.

We performed an intraoperative assessment of axillary lymph nodes by palpation and intraoperative ultrasound. Lymph nodes that appeared suspicious on clinical and/or intraoperative ultrasound were excised even if they had not captured radioactivity or had not stained blue with vital stain. We used this method in all the patients in our group.

Lymph nodes excised by any of these methods were sent to the pathology laboratory accompanied by patient data.

Sampling requires establishing the number of lymph nodes received per case, determining those that stained blue, those that were identified only by radioactive substances (sent in separate containers), and those which were identified only by palpation associated with intraoperative ultrasound evaluation, considered non-sentinel (non-SLN) and sent in a separate container, sectioning them at every 2 $\mathrm{mm}$ in parallel with the long axis, and embedding all fragments in paraffin. The fragments were then sectioned at 200 microns (in order to identify not only macro-, but also micrometastases) and a standard section was made at every interval (stained with hematoxylin-eosin), and a separate section was mounted and prepared for any subsequent immunohistochemical examination. We performed a comparative study of the total number of sentinel nodes excised by the 2 methods (radioactive versus combined), we also noted the number of positive lymph nodes (metastases) identified histologically by the 2 methods, and especially the existence of understaged patients following the use of a single method. The same parameters were also observed in non-SLN cases. Data were retrieved from histological reports and were analyzed statistically using Graph Pad Prism 6 software, Fisher test, chi square test, and T-test. p $<0.05$ was considered statistically significant.

\section{RESULTS}

The study included 69 patients initially. In 2 cases, the SLN could not be identified by any of the methods used and described in the Materials and methods part. These two cases required evaluation lymphadenectomy.

In the remaining 67 cases, both the radioactive and combined method (vital stain and radioactive) were applied simultaneously. Non-SLN, which were identified intraoperatively, were excised together with the sentinel nodes (23 cases).

The tracer technique was successfully performed in 67 cases $(97.1 \%)$ and the vital stain method in 33 cases (47.82\%), with a statistically significant difference, $\mathrm{p}=$ 0.0001 . Considerable differences were obtained in terms of the number of SLN detected by the two methods (radioactive versus vital stain). We identified a total number of 153 SLN (all showing obvious radioactivity), of these only 56 were stained with vital stain $(\mathrm{p}<0.0001)$. It should be noted that all blue stained sentinel lymph nodes showed radioactivity.

The mean number of sentinel nodes identified with methylene blue was 0.82 /patient, and 2.28 by the radioactive tracer method.

Twenty-two metastatic SLN were identified (14.37\%), which represented a percentage of $20.89 \%$ of patients. There is a significant difference between the two methods in this respect as well, thus, of the detected 22 metastatic SLN 22 were radioactive and only 5 stained with methylene blue ( $\mathrm{p}=0.0049)$.

During surgery, of the 67 cases 35 lymph nodes were excised, which had not captured the radionuclide and had not stained but were hypertrophied on palpation or intraoperative axillary ultrasound; they were categorized as non-sentinel lymph nodes ( 23 cases). Of these 35 nonSLN microscopic examination revealed 3 lymph nodes with metastases, and in 1 case metastases were found exclusively in the non-SLN (Table 1).

Of the patients with axillary metastases, 14 were identified by radionuclide, 5 by injecting vital stain, and 1 using palpation and intraoperative ultrasound (non-sentinel). The radionuclide method showed a higher sensitivity 
TABLE 1. Comparative analysis of the study group by applying different methods to identify SLN and non-SLN

\begin{tabular}{|c|c|c|c|c|c|}
\hline & $\begin{array}{l}\text { Radioactive method } \\
\text { No. of cases / } \\
\text { no. of SLN }\end{array}$ & $\begin{array}{c}\text { Vital stain method } \\
\text { No. of cases / } \\
\text { no. of SLN }\end{array}$ & $\begin{array}{c}\text { Combined method } \\
\text { No. of cases / } \\
\text { no. of SLN }\end{array}$ & $\begin{array}{c}\text { Non-sentinel LN } \\
\text { identification } \\
\text { method } \\
\text { No. of cases / } \\
\text { no. of SLN }\end{array}$ & $p$ value \\
\hline Positive & $14 / 22$ & $5 / 5$ & $14 / 22$ & $3 / 3$ & 0.1 \\
\hline Negative & $53 / 131$ & $62 / 51$ & $53 / 22$ & $20 / 32$ & \\
\hline Total & $67 / 153$ & $67 / 56$ & $67 / 153$ & $23 / 35$ & \\
\hline
\end{tabular}

(93.33\%) than the methylene blue method (33.33\%), but both had $100 \%$ specificity in detecting sentinel lymph nodes.

\section{DISCUSSIONS}

SLN biopsy has become the standard initial therapy in early forms of breast cancer, and lately also in the more advanced ones (following chemotherapy) with complete axillary response on radiologic examination. ${ }^{3,6}$ The classic technique involved injecting a vital stain in combination with radionuclide for a higher efficiency and accuracy. The difficulties in using a combined method are mostly related to the costs of the radiodetection equipment and to the availability of nuclear medicine services. Efforts were made to find less expensive and simpler solutions for the detection of sentinel lymph nodes through the exclusive use of the vital stain or by using magnetic powders. ${ }^{8,9}$

Moreover, researchers raised the question whether the exclusive use of radionuclide has the same accuracy and certainty as the combined method, because using the vital dye may give rise to adverse effects such as allergies or even skin necrosis.

Our nonrandomized prospective study mainly aims at comparing the efficiency of the two methods and the combined one.

SLN detection by the radionuclide method had a far superior detection rate compared to the vital stain method, practically in only $1 \%$ ( 2 cases) SLN could not be identified, and required assessment lymphadenectomy. Our study shows that standalone use of the vital stain may be effective in under $50 \%$ of cases.

We noted significant differences in the number of SLN detected by the two methods in favor of the radiotracer method. Detecting a real or complete number of sentinel lymph nodes is of great importance especially in light of new data and trends in world literature. Thus, in order to correctly apply the ACOSOGO Z00011 protocol, we need to have as much data on the lymph nodes as possible. The
Protocol provides for the avoidance of lymphadenectomy in patients with one or two positive nodes fulfilling five other criteria (primary tumor stage 1 or 2 , surgical preservation of the breast followed by postoperative radiotherapy on the remaining breast tissue, and the absence of chemotherapy or hormone therapy as neoadjuvant treatment) ${ }^{4}$ In the exclusive use of the vital stain, according to data drawn from the study, implementing the Z00011 protocol is impossible or perilous. Furthermore, the 2015 NCCN and ESMO protocols provide for the application of SLN biopsy in patients with preoperative neoadjuvant treatment (even with lymphadenopathy at the onset), but with complete axillary response on radiography. ${ }^{4,6}$ In these cases, the presence of axillary metastases is followed by lymphadenectomy. Accurate assessment of axillary cases with neoadjuvant treatment implies the excision of at least 3 SLN. ${ }^{6}$ This is actually impossible if only the vital stain is used, because our study revealed that the methylene blue method identified only $0.83 \mathrm{lymph}$ nodes/patient versus 2.28 lymph nodes/patient identified by the radionuclide method.

Another objective of the study was to assess the efficiency of parallel use of the palpation method and/or intraoperative ultrasound evaluation of suspicious axillary lymph nodes. This technique has been promoted as effective in the intraoperative evaluation of the axilla by other studies. ${ }^{14}$ In our study, the use of this method helped us to identify 35 non-SLN, of which, following microscopic examination, 3 lymph nodes had metastases, and in 1 case out of the 67 metastases were found exclusively in the nonSLN.

\section{CONCLUSION}

Given the superior efficiency of sentinel node detection by injecting radionuclide, as well as the risk of understaging by the vital stain method, we believe that the radioactive method should be used. The combination with the vital stain method is a choice only during the learning curve. 
Our study identified 35 non-sentinel lymph nodes of which 3 had metastases and in one case the metastases were located exclusively in the non-sentinel lymph node. As such, we can conclude that using the radioactive method combined with the intraoperative evaluation of the suspicious lymph nodes is of paramount importance.

\section{CONFLICT OF INTEREST}

Nothing to declare.

\section{ACKNOWLEDGEMENT}

This study was funded by the University of Medicine and Pharmacy of Tîrgu Mureș, Research Grant No. 13/2015.

\section{REFERENCES}

1. DeSantis CE, Bray F, Ferlay J, Lortet-Tieulent J, Anderson BO, Jemal A. International Variation in Female Breast Cancer Incidence and Mortality Rates. Cancer Epidemiol Biomarkers Prev. 2015;24(10):1495-1506.

2. Coates AS, Winer EP, Goldhirsch A. Tailoring therapies - improving the management of early breast cancer: St Gallen International Expert Consensus on the Primary Therapy of Early Breast Cancer 2015. Ann Oncol. 2015;26(8):1533-1546.

3. Ferrari A, Rovera F, Dionigi P, et al. Sentinel lymph node biopsy as the new standard of care in the surgical treatment for breast cancer. Expert Rev Anticancer Ther. 2006;6(10):1503-1515.
4. Giuliano AE, Hunt KK, Ballman KV, et al. Axillary Dissection vs No Axillary Dissection in Women With Invasive Breast Cancer and Sentinel Node Metastasis A Randomized Clinical Trial. JAMA. 2011;305(6):569-575.

5. Donker M, van Tienhoven G, Straver ME, et al. Radiotherapy or surgery of the axilla after a positive sentinel node in breast cancer (EORTC 1098122023 AMAROS): a randomised, multicentre, open-label, phase 3 noninferiority trial. Lancet Oncol. 2014;15(12):1303-1310.

6. Mocellin S, Goldin E, Marchet A, Nitti D. Sentinel node biopsy performance after neoadjuvant chemotherapy in locally advanced breast cancer: A systematic review and meta-analysis. Int J Cancer. 2016;138(2):472-480.

7. Zengel B, Yararbas U, Sirinocak A, et al. Sentinel lymph node biopsy in breast cancer: review on various methodological approaches. Tumori. 2013;99(2):149- 53

8. Asoglu $\mathrm{O}$, Ozmen $\mathrm{V}$, Karanlik $\mathrm{H}$, et al. The role of sentinel lymph node biopsy with blue dye alone in breast cancer patients with excisional biopsy. Acta Chir Belg. 2005;105(3):291-296.

9. Thill M, Kurylcio A2, Welter R. The Central-European SentiMag study: Sentinel lymph node biopsy with superparamagnetic iron oxide (SPIO) vs. radioisotope. Breast. 2014;23(2):175-179.

10. Bakhtiar N, Jaleel F, Moosa FA, Qureshi NA, Jawaid M. Sentinel lymph node identification by blue dye in patients with breast carcinoma. Pak J Med Sci. 2016;32(2):448-451.

11. O'Reilly EA, Prichard RS, Al Azawi D, et al. The Value of Isosulfan Blue Dye in Addition to Isotope Scanning in the Identification of the Sentinel Lymph Node in Breast Cancer Patients With a Positive Lymphoscintigraphy: A Randomized Controlled Trial (ISRCTN98849733). Ann Surg. 2015;262(2):243-248

12. Kern KA. Concordance and validation study of sentinel lymph node biopsy for breast cancer using subareolar injection of blue dye and technetium 99m sulfur colloid. J Am Coll Surg. 2002;195(4):467-475.

13. Garbay JR, Skalli-Chrisostome D, Leymarie N, Sarfati B, Rimareix F, Mazouni C. The Role of Blue Dye in Sentinel Node Detection for Breast Cancer: A Retrospective Study of 203 Patients. Breast Care (Basel). 2016;11(2):128-132.

14. V Klimberg, C Townsend. Atlas of Breast Surgical Techniques. 1st Edition. Ed. SaundersPrint Book. ISBN :9781416046912, 2009. 